\title{
Early post-induction Augmented therapy improves outcome in children and adolescents with T-cell acute lymphoblastic leukemia
}

\author{
Mayada Abu Shanap ${ }^{1}$, Haytham Aljbour ${ }^{1}$, Rawad Rihani ${ }^{1}$, Hasan Hashem ${ }^{1}$, Amal Abu \\ Ghosh $^{1}$, Abdelghani Tbakhi ${ }^{1}$, Nazmi Kamal ${ }^{2}$, Iyad Sultan ${ }^{1}$, and Faris Madanat ${ }^{1}$ \\ ${ }^{1}$ King Hussein Cancer Center \\ ${ }^{2}$ King Hussien Medical Center
}

September 25, 2021

\begin{abstract}
Introduction: T-cell acute lymphoblastic leukemia (T-ALL) accounts for approximately $15 \%$ of all newly diagnosed ALL in children and adolescents and is associated with worse outcomes compared to pre-B ALL. We aimed to decrease T-ALL relapses by intensifying our regimen. Methods: Patients with T-ALL were treated using two different regimens; before September 2014, patients were treated per St. Jude Total XV protocol; subsequently, a major change was adopted by adding two intensive blocks: FLAG and Re-Intensification (fludarabine, dexamethasone, cytarabine, etoposide and asparaginase). Cranial radiation was limited to patients with $\mathrm{WBC}>=100 \mathrm{k} / \mu \mathrm{l}$ at diagnosis and/or patients with CNS2/CNS3 status. Results: Between June 2005 and April 2020, a total of 100 patients (76 males) were treated and followed for a median of 70 months (range, 14-181 months). Median age at diagnosis was 9 years (range,0.5-17.8 years). Forty-eight patients were diagnosed after September 2014 and received the augmented regimen; their median follow up was 46 months (range,14-74 months). The 5-yr-EFS estimates for patients who received the augmented regimen vs. standard regimen were $87 \% \pm 4.9 \%$ vs $67 \% \pm 6.8 \%$ ( $p=0.03$ ); and the 5 -yr-OS estimates were $87 \% \pm 5.1 \%$ vs. $71 \% \pm 6.3 \%(\mathrm{p}=0.06)$ respectively. Treatment related mortality (TRM) were reported in 2 patients treated using our standard regimen but none for patients who received the augmented regimen. Conclusions: We implemented a novel approach with early intensification added to a backbone of modified St. Jude Total-XV regimen for patients with T-ALL that resulted in improved outcome with no treatment related mortality.
\end{abstract}

\section{Introduction:}

T-cell acute lymphoblastic leukemia (T-ALL) accounts for $15 \%$ of newly diagnosed cases of childhood acute lymphoblastic leukemia (ALL). Historically, T-ALL has had inferior event-free survival (EFS) and overall survival (OS) compared with precursor B-cell ALL (B-ALL). Patients with T-ALL often present with unfavorable clinical features, including older age, higher white blood cell count (Wbc), and extramedullary disease, especially in the central nervous system. Additionally, T-ALL was reported to have increased risk of induction failure. [1-4]

Outcomes for T-ALL have improved with contemporary risk and response-based treatment protocols: Early intensified therapy, with a 4-drug induction containing dexamethasone and an anthracycline followed by augmented BFM-like consolidation containing cyclophosphamide, asparaginase and methotrexate (MTX), but relapse rates remain higher and relapses often unsalvageable. [5-13]

The outcome of children and adolescents with T-ALL treated at our center per modified St. Jude total XV regimen was reported in 2014; the 5-year-OS and EFS for Early T-cell precursor acute lymphoblastic leukemia (ETP)were $77.1 \%$ and $79.6 \%$; while for non-ETP, these estimates were $78.5 \%$ and $65.2 \%$, respectively.[14]

In an attempt to improve the outcome of our patients we modified our regimen by using an early intensive 
post-induction chemotherapy composed of fludarabine, high dose cytarabine and etoposide. This approach was inspired by the augmented therapy in European protocols for high-risk leukemia. [7, 15]

We report here our results using both regimens (standard and augmented) in children and adolescents who presented to our center with untreated T-ALL over a period of over 16 years.

\section{Methods:}

\section{Patients}

After obtaining approval of our institutional review board (IRB \#:19KHCC34), we retrospectively reviewed all patients who presented with T-ALL to our department from January 2005 until April 2020. Patients' records were identified by querying our departmental database. All patients with T-ALL presented to our institution and treated per modified St Jude Total VX protocol; we excluded patients who presented with relapsed disease, received previous therapy before referral and patients who underwent hematopoietic cell transplantation (HCT) in first remission.

\section{Treatment:}

Standard protocol:

All T-ALL patients were treated per modified St Jude total XV protocol. Treatment consisted of remission induction, consolidation, and continuation. Remission induction comprised of dexamethasone, vincristine, daunorubicin, and asparaginase. Patients with a level of minimal residual disease (MRD) of $0.1 \%$ or more on day 15-19 received three additional doses of asparaginase or one dose of peg asparaginase. Subsequent induction therapy consisted of cyclophosphamide, mercaptopurine, and cytarabine (Appendix Table 1A). On hematopoietic recovery (between days 43 and 46), the minimal residual disease was assessed, and consolidation therapy was begun with four doses of High dose methotrexate (HD-MTX) at dose of $5 \mathrm{gm} / \mathrm{m} 2$ and daily mercaptopurine (Appendix Table 1C). During initial continuation therapy, patients received intensive schedule of asparaginase chemotherapy, daily mercaptopurine interrupted with pulses of doxorubicin plus vincristine plus dexamethasone and two reinduction cycles, followed by three rotating drug pairs. Continuation treatment lasted 120 weeks in girls and 146 weeks in boys (Appendix Table 1E).

Augmented regimen:

In September 2014, we modified our regimen for T-ALL by adding two blocks of chemotherapy to all patients; including FLAG and re-intensification. FLAG as (fludarabine $15 \mathrm{mg} / \mathrm{m} 2 \times 4$ days, high dose cytarabine as 2 $\mathrm{gm} / \mathrm{m} 2 \mathrm{X} 4$ days) in patients with negative MRD or MRD $>=0.01 \%$ and $<1 \%$, and FLAG as (fludarabine 30 $\mathrm{mg} / \mathrm{m} 2 \times 4$, high dose Cytarabine as $2 \mathrm{gm} / \mathrm{m} 2 \mathrm{X} 4$ ) if $\mathrm{MRD}>=1 \%$ (Appendix Table 1B). Re-Intensification (high dose cytarabine, dexamethasone, VP16, peg-asparaginase) was given to all patients (Appendix Table 1D); re-induction II was omitted in the augmented therapy group (Appendix Table 1E).

CNS directed therapy:

All patients received triple intrathecal therapy (IT) on days 1 and 15 of induction with additional doses on days 8 and 22 in patients with peripheral blood leukocyte count $(\mathrm{Wbc})>=50,000 / \mu \mathrm{L}$ at presentation and continued through the consolidation and continuation therapy (total number,21-23). Cranial radiation (CRT) was given to patients at increased risk of CNS relapse, including patients with Wbc count $>=100,000 / \mu \mathrm{L}$ at presentation (CRT dose of 12Gy) and patients with CNS-2 or CNS-3 status at diagnosis or those who had a traumatic lumbar puncture with blasts (CRT dose of 18Gy).

\section{Definitions and statistical analysis}

According to our clinical practice guidelines which were drafted in 2005, failure of induction is defined as $\mathrm{MRD}>=1 \%$ at day 35-42 of remission induction. Refractory disease (RD) was defined as leukemia persistence in patients surviving induction. Relapse was defined as disease recurrence at any site after achieving remission. Treatment-related mortality (TRM) was defined as death in remission in patients receiving treatment for ALL. Rapid early (RER) was defined as M1 marrow ( $<5 \%$ blasts) on day 15, and 
M1 marrow with negative MRD status $(<0.01 \%)$ at the end of Induction. Slow early responder (SER) M2 $(5-25 \%$ blasts $)$ or M3 ( $>25 \%$ blasts) marrow on Day 15 or positive MRD status $(>=0.01 \%)$ at end of induction.

Overall survival (OS) was measured from the time of entry in the protocol to the time of death by any cause or last follow-up. Event-free survival (EFS) was defined from the time of entry in the protocol to the date of relapse or date of refractory disease post FLAG and/or re-intensification chemotherapy, date of second cancer or death from any cause or date of last follow up. Data analysis was performed using R software (V4.0.4.) with calculation of Kaplan Meier estimates and log-rank tests using "survival" package and drawing survival curves using "survminer" package. We iterated through all possible covariates to calculate the p-values using log-rank test. Significant covariates were included in a multivariate Cox-regression model. Chi-square test and Mann-Whitney test were used to evaluate categorical and numeric variables, respectively. A p-value of $=<0.05$ was considered significant.

\section{Results:}

\section{Patients characteristics}

One hundred patients were included in this study; median age at diagnosis was 9.2-years (range, 0.5-17.8 year); $76 \%$ were males; 30 patients $(30 \%)$ presented with initial WBC count [?]200 $\times 10^{9} / \mathrm{L}$; the majority of the patients $(76 \%)$ had high risk disease per NCI classification. CNS involvement was documented in 15 patients (15\%). There were no significant differences in the distributions of age, sex, leukocyte count, CNS status at diagnosis and NCI risk between the 2 groups (Table 1 ).

Response to induction therapy:

Sixty-eight $(68 \%)$ patients had rapid early response. MRD evaluation at day 15 showed $>=0.01 \%$ involvement in $37 \%$ and $63 \%(\mathrm{p}=0.0009)$ of patients in standard and augmented groups, respectively. Similarly, the end of induction MRD was $>=0.01 \%$ in $8 \%$ and $23 \%(\mathrm{p}=0.03)$ of patients in standard and augmented groups, respectively.

\section{Outcomes and prognostic factors:}

At a median follow up of 70 months (range, 14 to 181 months), the 5 -year EFS was $76 \% \pm 4.4 \%$ and the 5 -year OS was $78 \% \pm 4.2 \%$ for the whole group. When compared using log-rank test, the 5-year EFS for patients treated in the augmented therapy group was $87 \% \pm 4.9 \%$ which is significantly better than the standard therapy group estimate of $67 \% \pm 6.5 \%(\mathrm{p}=0.03)$ (Figure.1A). However, there was no statistically significant difference in 5 -year OS between the 2 groups $87 \% \pm 5.1 \%$ in augmented vs. $71 \% \pm 6.2 \%$ in standard group, $\mathrm{p}=0.061)$ (Figure.1B)

In a univariate analysis model, other factors associated with better EFS and OS were: Response to therapy at day 15 of remission induction and cranial radiation (Appendix Table 2).

Notably, none of the patients $(\mathrm{N}=21)$ who received CRT relapsed in both group; the 5-year Relapse free survival (RFS) in patients who received CRT in both groups was $100 \% \pm 0 \%$, and in patients who did not receive CRT in augmented and standard therapy group was $(94 \% \pm 4.2 \%, 62 \% \pm 7.7 \%, \mathrm{P}=0.0004)$, respectively (Figure 2).

In total 79 patients did not received CRT ( $N=41$ in standard therapy, $\mathrm{N}=38$ in augmented therapy). Seventeen $(22 \%)$ of 79 patients who did not receive CRT had bone marrow or isolated extramedullary relapse. Among these 17 patients, 15/79(19\%) were treated in standard therapy group and 2/79 (3\%) in the augmented therapy group. (Table 2)

\section{Toxicity}

Regarding hematological toxicities all patients in the augmented therapy group were admitted with febrile neutropenia post each block of intensive therapy, seven of 48 patients needed intensive care and another 
four patients had invasive fungal infections, all are alive. Notably, all had grade 3 anemia, and grade 34 thrombocytopenia; the toxic effects of augmented therapy were considerable but manageable with no treatment related death. Non- Hematological toxicities between two groups were comparable.

Events:

There were 17 events in the standard therapy group (15 relapses, and 2 treatment related death due to liver failure and sepsis), while patients treated in the augmented therapy group had 6 events (one died of second cancer/ AML, 2 relapsed, and 3 had refractory disease). The median time to relapse for all patients was 13.8 months (range, 6-55 months). Eleven of 17 patients who relapsed (65\%) had isolated BM relapse, 4 had isolated extramedullary relapse, and 2 had combined relapse (BM+CNS). Six patients (35\%) achieved CR2 after salvage chemotherapy, of them three patients underwent allogenic -HCT in CR2, only one patient alive in continuous complete remission $(\mathrm{CCR})$ post-transplant; all others $(\mathrm{N}=5)$ succumbed due to second relapses.

\section{Discussion:}

The outcome of children with T-ALL improved markedly; we previously reported the outcome of T-ALL treated at KHCC per St. Jude total XV protocol [14].In September 2014 we adopted a new protocol of augmented post-induction therapy in all patients with T-ALL treated at our center regardless of the MRD at end of induction. The rationale for this approach is early intensification of therapy post induction to clear any residual MRD and decrease the relapse when the MRD measurement at day 15 and end of induction may not be successful in identifying certain groups who are still MRD positive but not detectable by our current techniques. Patients treated per St. Jude total XV already exposed dexamethasone, AIEOP-BFM consolidation /protocol IB that includes cyclophosphamide, cytarabine and 6- mercaptopurine at week 4 and 5 of remission Induction[16]; and intensive asparaginase therapy in early continuation[11]. So, we adopted different agents for intensification of therapy; fludarabine, high dose cytarabine, VP16, dexamethasone, pegasparaginase; combination has been used and proven to be effective in refractory relapsed ALL [17, 18].

Our results showed significant improvement of EFS in patients treated using augmented therapy $87 \%$ vs $67 \%$ in standard therapy group. Notably, 11 patients in the augmented group had positive MRD $(>=0.01 \%)$ and/or M2, M3 marrow at the end of induction. Eight (73\%) of these patients achieved remission with negative MRD $(<0.01 \%)$ post FLAG chemotherapy, 7 of them remained in CCR at date of last follow-up.

Attempts to intensify treatment of patients with T-ALL are well-reported in the literature; our results are in line with outcomes reported by other contemporary clinical trials of T-ALL in children and adolescent. BFM Augmented post-induction chemotherapy results in an excellent outcome for T-ALL, event-free survival at three years was $92 \%$ in the augmented-therapy group and $71 \%$ in the standard-therapy group[16]. Consolidation phase IB used in AIEOP-BFM protocols, based on cyclophosphamide, 6-mercaptopurine, and ara-C at conventional (non-high) doses was shown to be effective in reducing MRD in Early T-cell precursor ALL; with 3-year event-free survival of $86 \%$, which is not different from that of the other T-cell acute lymphoblastic leukemia variants [19].

Ajay Vora et al reported improved outcome for Children and Young Adults with T-ALL treated with a regimen that included Pegylated E. Coli Asparaginase (Peg-ASP) (Oncospar, Medac UK) and Dexamethasone (UKALL 2003 regimen). EFS and OS were significantly better for T-ALL in UKALL 2003 (3 year: EFS 86\%, OS 90\%) in comparison with a previous regimen- ALL97/99 (3-year EFS 73\%, OS 78\%)[20].

Dana Farber Cancer Institute Consortium (DFCI) 85-01 protocols demonstrated superior outcomes in TALL patients treated with intensive consolidation regimens that included 30 weeks of ASP. Patients with HR received every 3 -wk cycles with vincristine, dexamethasone, 6MP and doxorubicin. The 4-yr EFS and OS for all T-ALL pts was $83 \%$ and $89 \%$, respectively [21].

In NOPHO 2008, MRD based chemotherapy with intensive three blocks of chemotherapy for T-ALL with MRD [?]0.1\% on day 29, led to favorable outcomes; The 5-year event-free survival was $74 \%$ [19]. 
In our cohort, patients who had rapid early response with M1 marrow at day 15 of remission induction were at lower risk of relapse when compared with T-ALL with M2/M3 marrow $(\mathrm{p}=0.011)$. Relapse rate in T-ALL patients with positive MRD at end of induction was 6/15 (40\%), while14 patients (17\%) of 85 patients with negative MRD relapsed $(\mathrm{p}=0.06)$. While the later MRD timepoint most effectively identifies high risk patients, the earlier end-of-Induction time point can be used to identify lower risk patients who can safely receive less intensive therapy. In the UKALL2003 trial, T-ALL patients with end-of-Induction MRD $<10-4$ received standard BFM consolidation with a standard interim maintenance phase instead of Capizzi escalating MTX with asparaginase and had a 5yr EFS of 93.1\% [22, 23].

Markedly elevated white blood cell count (Wbc)[?]200 in our cohort was not associated with inferior outcome when compared with patients presented with WBC $<200$ (EFS p=0.7), and there was no difference in outcome at age threshold of 10 years (EFS $\mathrm{p}=0.4$ ).

In our study, none of the patients who received CRT in both groups $(\mathrm{N}=21)$ relapsed while 17 of $73(23 \%)$ patients who did not receive CRT relapsed. Interestingly only 2 of the augmented group patients who did not receive CRT relapsed. This suggests that systemic augmentation may compensate for the lack of CRT. This additional benefit should be taken in consideration, especially when treating young children.

Currently, approaches to the use of CRT for pediatric T-ALL are variable, with some cooperative groups administering CRT to all T-cell patients, some omitting CRT in all patients, and some using a risk-stratified approach.[24]

In Total XV, no patient received prophylactic cranial radiation, but additional intrathecal therapy during early induction, intensification with asparaginase, HD-MTX, and dexamethasone seemed to contribute to improved CNS control; the rate of CNS relapses in T-ALL (7.9\%) that was equivalent to that observed on the predecessor study, in which CRT was used in selected high-risk patients with T-ALL [11]

In Total XVI, the cumulative incidence (CI) of CNS relapse in T-ALL was (Any or Isolated is 4.3\%). Only T-ALL retained independent significance in the multivariable analysis for any CNS relapse (hazard ratio, 5.15 [95\% CI, 1.3 to 20.6]; P = 0. 021) and for isolated CNS relapse (hazard ratio, 6.83 [95\% CI, 1.5 to 30.5]; $\mathrm{P}=.012)[6]$

The European Organization for the Research and Treatment of Cancer (EORTC) has eliminated CRT in all patients with T-ALL, including those with CNS disease, in its recent studies and has adopted regimens with an intensified schedule of high-dose MTX (HD-MTX) and triple intrathecal chemotherapy (MTX, cytarabine, and hydrocortisone). With this approach, isolated and overall CNS relapse rates of $5.3 \%$ to $8.5 \%$ were observed on study 5895 . [8, 15, 25]

Excellent outcomes with 5-year EFS of $81.2 \%$ and low rates of isolated CNS relapse (3.5\%) were also reported for T-ALL on the UKALL 2003 trial with a regimen that did not include either HD-MTX or prophylactic CRT. [26, 27]

The COG AALL0434 trial treated patients with T-ALL on an augmented BFM regimen and randomly assigned patients to receive either HD-MTX with leucovorin rescue or escalating methotrexate without leucovorin (Capizzi) and Nelarabine and cranial radiation; the cumulative incidence of central nervous system relapse was lower in patients who received Capizzi methotrexate and nelarabine. [28-30]

Our study has multiple limitations that adds to its retrospective design. First, small number of patients in each cohort and reduced events limit the power of our statistical analysis. Second, patients treated per augmented therapy need longer duration of follow up, to prove that excellent cure rate we have achieved is durable. Third, T- ALL patients treated after sep/2014 received intensive therapy by adding two blocks of chemotherapy regardless of MRD response after remission induction due limitation in our MRD study in T-ALL, 37 patients (77\%) of 48 in augmented therapy group received FLAG consolidation despite having a negative MRD $(<0.01 \%)$ at end of induction; the objective of early intensification therapy is to prevent unsalvageable relapses which is relatively balanced with treatment burden. The toxic effects of augmented therapy were considerable but manageable. Hematological toxicities are balanced against no increase in 
therapy related mortality as supportive care improvement over time may also impacts favorable outcomes; the mean total duration of hospitalization was slightly longer for patients in the augmented-therapy group than in the standard-therapy group.

In summary, we showed that early intensification of systemic and CNS-directed chemotherapy can result in an improvement of outcome in T-ALL patients as it significantly decreases risk of relapse. Response-based de-escalation of therapy and Escalation of therapy based on MRD at later time point is highly needed as this could limit unnecessary intensification and reduce treatment-related mortality and morbidity. Additionally; we showed that the rate of relapse in patients who did not receive CRT was significantly lower in patients who were treated with our augmented therapy compared to standard therapy. This was likely related to effective intensive CNS directed therapy; omitting CRT in setting of augmented therapy is warranted. The use of prophylactic cranial radiation therapy in the treatment of patients with T-ALL is declining given the higher rates of neurocognitive sequelae, endocrinopathies, and secondary malignancies associated with CRT; several studies have now shown that CRT can be successfully eliminated from regimens that contain intensive systemic and intrathecal chemotherapy.

\section{Conflict of interest statements:}

The authors declare that there is no conflict of interest.

\section{References:}

1. Hunger, S.P., et al.,Improved survival for children and adolescents with acute lymphoblastic leukemia between 1990 and 2005: a report from the children's oncology group. J Clin Oncol, 2012. 30 (14): p. 1663-9.

2. Pui, C.H., et al., Childhood Acute Lymphoblastic Leukemia: Progress Through Collaboration. J Clin Oncol, 2015. 33 (27): p. 2938-48.

3. Steinherz, P.G., et al., Treatment of patients with acute lymphoblastic leukemia with bulky extramedullary disease and T-cell phenotype or other poor prognostic features: randomized controlled trial from the Children's Cancer Group.Cancer, 1998. 82 (3): p. 600-12.

4. Uckun, F.M., et al., Biology and treatment of childhood T-lineage acute lymphoblastic leukemia.Blood, 1998. 91 (3): p. 735-46.

5. Teachey, D.T. and D. O'Connor,How I treat newly diagnosed T-cell acute lymphoblastic leukemia and T-cell lymphoblastic lymphoma in children. Blood, 2020.135 (3): p. 159-166.

6. Jeha, S., et al., Improved CNS Control of Childhood Acute Lymphoblastic Leukemia Without Cranial Irradiation: St Jude Total Therapy Study 16. J Clin Oncol, 2019.37 (35): p. 3377-3391.

7. Quist-Paulsen, P., et al.,T-cell acute lymphoblastic leukemia in patients 1-45 years treated with the pediatric NOPHO ALL2008 protocol. Leukemia, 2020.34 (2): p. 347-357.

8. Hofmans, M., et al., Results of successive EORTC-CLG 58881 and 58951 trials in paediatric T-cell acute lymphoblastic leukaemia (ALL). Br J Haematol, 2019.186 (5): p. 741-753.

9. Moricke, A., et al.,Dexamethasone vs prednisone in induction treatment of pediatric ALL: results of the randomized trial AIEOP-BFM ALL 2000. Blood, 2016.127 (17): p. 2101-12.

10. Place, A.E., et al.,Intravenous pegylated asparaginase versus intramuscular native Escherichia coli $L$ asparaginase in newly diagnosed childhood acute lymphoblastic leukaemia (DFCI 05-001): a randomised, open-label phase 3 trial. Lancet Oncol, 2015. 16 (16): p. 1677-90.

11. Pui, C.H., et al., Treating childhood acute lymphoblastic leukemia without cranial irradiation. N Engl J Med, 2009. 360 (26): p. 2730-41.

12. 2014, P.A.A.M.A.

13. Winter SS blood 2015, A. 
14. Madanat, F., et al.,Features and outcomes of pediatric early T cell leukemia: King Hussein Cancer Center experience. Hematol Oncol Stem Cell Ther, 2016.9 (3): p. 126-8.

15. Vilmer, E., et al.,Long-term results of three randomized trials (58831, 58832, 58881) in childhood acute lymphoblastic leukemia: a CLCG-EORTC report. Children Leukemia Cooperative Group. Leukemia, 2000. 14 (12): p. 2257-66.

16. Nachman, J.B., et al.,Augmented post-induction therapy for children with high-risk acute lymphoblastic leukemia and a slow response to initial therapy. N Engl J Med, 1998. 338 (23): p. 1663-71.

17. Parker, C., et al.,Outcomes of patients with childhood B-cell precursor acute lymphoblastic leukaemia with late bone marrow relapses: long-term follow-up of the ALLR3 open-label randomised trial. Lancet Haematol, 2019. 6 (4): p. e204-e216.

18. Parker, C., et al., Effect of mitoxantrone on outcome of children with first relapse of acute lymphoblastic leukaemia (ALL R3): an open-label randomised trial.Lancet, 2010. 376 (9757): p. 2009-17.

19. Conter, V., et al., Early T-cell precursor acute lymphoblastic leukaemia in children treated in AIEOP centres with AIEOP-BFM protocols: a retrospective analysis.Lancet Haematol, 2016. 3 (2): p. e80-6.

20. Vora, A., et al., Improved outcome for children and young adults with T-cell acute lymphoblastic leukaemia (ALL): results of the United Kingdom Medical Research Council (MRC) Trial UKALL 2003. Blood, 2008. 112 (11): p. 908.

21. Place, A.E., et al.,Outcome of childhood T-cell acute lymphoblastic leukemia (T-ALL): Results from DFCI protocol 05-001 , 2014, American Society of Clinical Oncology.

22. Vora, A., et al., Augmented post-remission therapy for a minimal residual disease-defined high-risk subgroup of children and young people with clinical standard-risk and intermediate-risk acute lymphoblastic leukaemia (UKALL 2003): a randomised controlled trial. Lancet Oncol, 2014.15 (8): p. 809-18.

23. Vora, A., et al., Treatment reduction for children and young adults with low-risk acute lymphoblastic leukaemia defined by minimal residual disease (UKALL 2003): a randomised controlled trial. Lancet Oncol, 2013.14 (3): p. 199-209.

24. Kelly, M.J., et al.,Cranial radiation for pediatric T-lineage acute lymphoblastic leukemia: a systematic review and meta-analysis. Am J Hematol, 2014.89 (10): p. 992-7.

25. De Moerloose, B., et al.,Low CNS relapse incidence without radiotherapy and improvement of outcome: results of subsequent EORTC-CLG 58881 and 58951 trials in pediatric T-cell acute lymphoblastic leukemia (ALL) , 2012, American Society of Hematology.

26. Vora, A.J., et al., UKALL 2003, a randomised trial investigating treatment intensification for children and young adults with minimal residual disease defined high risk acute lymphoblastic leukaema, 2012, American Society of Hematology.

27. Patrick, K., et al.,Outcome for children and young people with Early T-cell precursor acute lymphoblastic leukaemia treated on a contemporary protocol, UKALL 2003. Br J Haematol, 2014. 166 (3): p. 421-4.

28. Dunsmore, K.P., et al.,Children's Oncology Group AALL0434: A Phase III Randomized Clinical Trial Testing Nelarabine in Newly Diagnosed T-Cell Acute Lymphoblastic Leukemia. J Clin Oncol, 2020. 38 (28): p. 3282-3293.

29. Winter, S.S., et al., Safe integration of nelarabine into intensive chemotherapy in newly diagnosed T-cell acute lymphoblastic leukemia: Children's Oncology Group Study AALL0434. Pediatr Blood Cancer, 2015. 62 (7): p. 1176-83.

30. Dunsmore, K.P., et al.,Pilot study of nelarabine in combination with intensive chemotherapy in high-risk T-cell acute lymphoblastic leukemia: a report from the Children's Oncology Group. J Clin Oncol, 2012.30 
(22): p. 2753-9.

Figure Legends:

Figure 1. Event-free survival (EFS)(A) and overall survival (OS) (B)of patients with T-acute lymphoblastic leukemia treated according per standard and augmented therapy groups.

Figure 2. Relapse free survival (RFS) in patients with T-acute lymphoblastic leukemia treated in standard and augmented therapy with or without cranial radiation (CRT).

Table legends:

TABLE 1. Characteristics of patients at diagnosis treated on Standard vs Augmented therapy and outcomes.

TABLE 2. Relapse in standard and augmented therapy group based on cranial radiation therapy.

Supporting Information file: Supplementary appendix
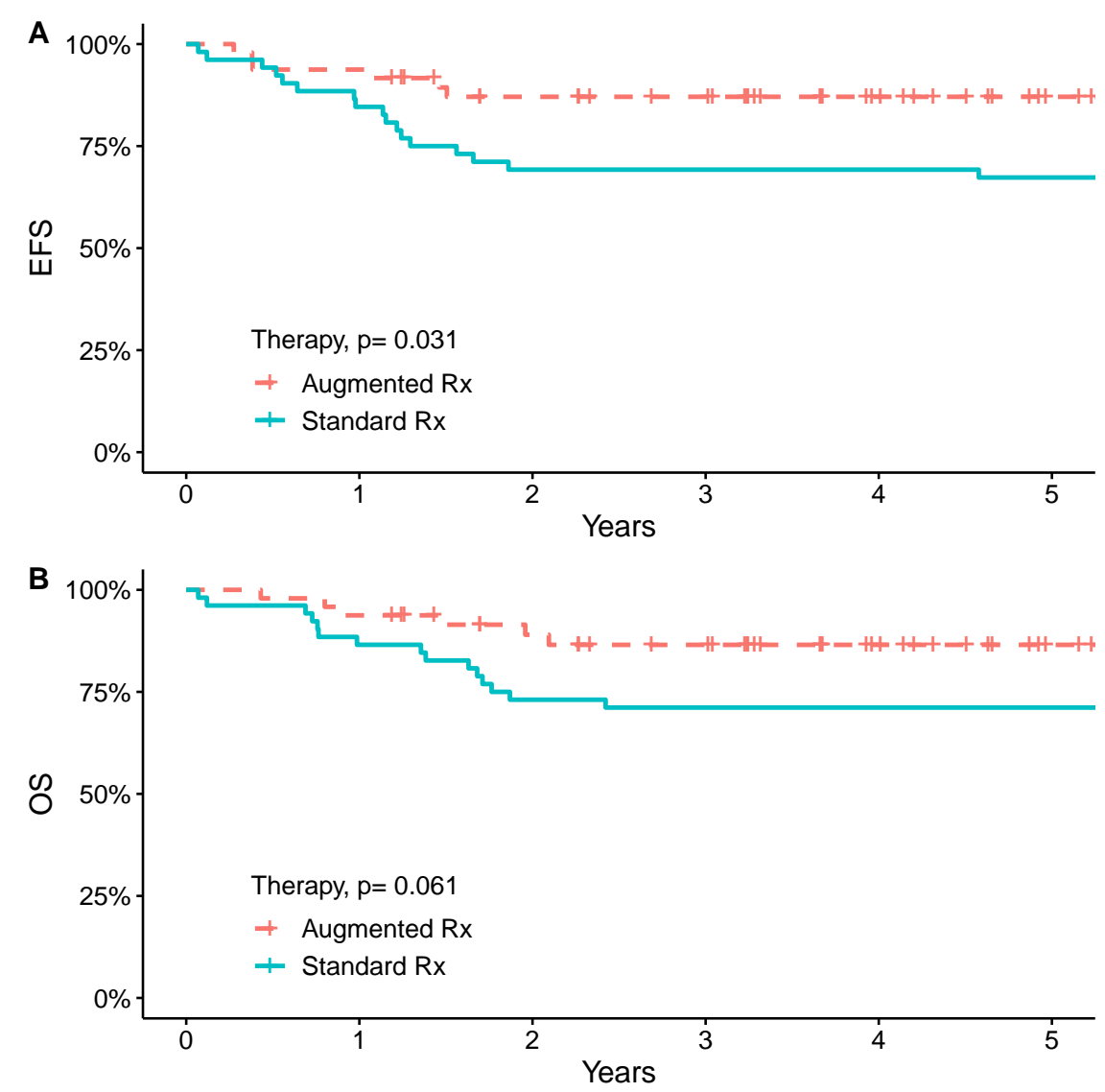




\section{Hosted file}

Table 1.docx available at https://authorea.com/users/393695/articles/539160-early-postinduction-augmented-therapy-improves-outcome-in-children-and-adolescents-with-t-cellacute-lymphoblastic-leukemia

\section{Hosted file}

Table 2.docx available at https://authorea.com/users/393695/articles/539160-early-postinduction-augmented-therapy-improves-outcome-in-children-and-adolescents-with-t-cellacute-lymphoblastic-leukemia 